\title{
Canine Responses to Hypoglycemia in Patients with Type 1 Diabetes
}

\author{
Deborah L. Wells, Ph.D., ${ }^{1}$ Shaun W. Lawson, Ph.D., ${ }^{2}$ and A. Niroshan Siriwardena, Ph.D. ${ }^{3}$
}

\begin{abstract}
Objective: Anecdotal evidence suggests that domestic dogs may be able to detect hypoglycemia in their human caregivers; scientific investigation of this phenomenon, however, is sorely lacking. This study thus aimed to investigate how pet dogs respond to the hypoglycemic episodes of their owners with type 1 diabetes.

Methods: Two hundred and twelve dog owners (64.2\% female) with medically diagnosed type 1 diabetes participated in the study. All participants owned at least $1 \mathrm{dog}$. Each person completed a purpose-designed questionnaire developed to collect information on their dogs' responses (if any) to their hypoglycemic episodes.

Results: One hundred and thirty-eight (65.1\%) respondents indicated that their dog had shown a behavioral reaction to at least one of their hypoglycemic episodes, with $31.9 \%$ of animals reacting to 11 or more events. Canine sex, age, breed status, and length of pet ownership were unrelated to hypoglycemia-response likelihood. Thirty-six percent of the sample believed that their dogs reacted most of the times they went "low"; $33.6 \%$ indicated that their pets reacted before they themselves were aware they were hypoglycemic. Dogs' behavioral responses to their owners' hypoglycemic episodes varied. Most animals behaved in a manner suggestive of attracting their owners' attention, for example, vocalizing (61.5\%), licking them (49.2\%), nuzzling them $(40.6 \%)$, jumping on top of them (30.4\%), and/or staring intently at their faces $(41.3 \%)$. A smaller proportion showed behavioral responses suggestive of fear, including trembling $(7.2 \%)$, running away from the owner $(5.1 \%)$, and/or hyperventilating (2.2\%).

Conclusions: The findings suggest that behavioral reactions to hypoglycemic episodes in pet owners with type 1 diabetes commonly occur in untrained dogs. Further research is now needed to elucidate the mechanism(s) that dogs use to perform this feat.
\end{abstract}

\section{Introduction}

$\mathbf{P}$ et-keeping is a widespread and well-accepted phenomenon in today's society. As a nation of self-confessed "animal lovers," the British public now share their homes with over 9 million cats and 6 million dogs. ${ }^{1}$ These animals can play an enormous role in their owners' lives. As well as providing a source of companionship, emotional support, and entertainment, there is now substantial evidence to suggest that domestic pets may be able to promote their owners' health. ${ }^{2-6}$

Lately, some attention has been directed toward the ability of companion animals, particularly dogs, to serve as an "early warning system" for certain types of underlying physical ailments in humans. For example, dogs have been shown to be capable of using olfaction to detect the presence of can- cer. In 1989, Williams and Pembroke ${ }^{7}$ reported a case of a Border Collie/Doberman Pinscher crossbreed sniffing repeatedly at a mole on its owner's leg that later was found to be malignant. Similar anecdotal reports have since appeared in newspapers and scientific journals. ${ }^{8-10}$ More recently, Willis and colleagues ${ }^{11}$ successfully trained 6 dogs of mixed breed to identify people with bladder cancer using a discrimination task. As a group, the dogs correctly identified urine samples from patients with bladder cancer on 22 of 54 occasions; a mean success rate of $41 \%$. Although this proof of principle study has been criticized for methodological weakness and erroneous conclusions, ${ }^{12}$ other work, using different methods, has yielded similar results. ${ }^{13,14}$

In addition to detecting cancer, there is now mounting evidence to suggest that some dogs can reliably predict the on-

${ }^{1}$ School of Psychology, Queen's University Belfast, Belfast, Northern Ireland, United Kingdom.

${ }^{2}$ Department of Computing and Informatics, University of Lincoln, Brayford Pool, Lincoln, United Kingdom.

${ }^{3}$ School of Health and Social Care, University of Lincoln, Lincoln, United Kingdom. 
set of epileptic seizures in their human owners, in some cases up to $45 \mathrm{~min}$ in advance of an event. ${ }^{15-19}$ This ability appears to be inherent in some animals. However, many dogs are now being trained by specialist organizations (e.g., Support Dogs, UK) to monitor their owners for outward signs of an imminent seizure and to react in an appropriate manner (e.g., barking or pawing) if a seizure is predicted. $15,16,18-20$

Hypoglycemia is a common and potentially hazardous complication of diabetes, usually occurring in individuals with type 1 (and increasingly type 2 ) diabetes who are receiving insulin. The condition typically results in physiological effects arising from the attempt to restore glucose levels (sympatheticoadrenal response). These effects can include fear, tremor, tachycardia, and/or sweating, as well as a critical lack of glucose to nervous tissue (neuroglycopenia), leading to behavioral change, seizures, or coma. ${ }^{21}$

Despite the wide range of hypoglycemia-related signals, people with diabetes often experience poor awareness of their own symptoms, with accuracy of detection typically waning over time due to the effects of advancing age or complications of the condition, such as autonomic neuropathy. ${ }^{22}$ Although numerous techniques (e.g., glucose sensors, skin conductors, heart-rate monitors) have been developed to facilitate early detection of hypoglycemia, ${ }^{23,24}$ none of these, as yet, are considered reliable, practical, or suitable for use outside clinical settings.

Anecdotal evidence now suggests that some dogs may be able to detect a fall in their owner's glucose levels. Lim and associates, $^{25}$ for example, indicated that 14 of 37 dog owners with diabetes recognized a response from their pet in reaction to their hypoglycemic episodes. A small number of case reports have also shown that dogs may be able to warn owners of impending hypoglycemia before symptoms are noticed by those whose awareness of the condition is mostly intact. ${ }^{26,27}$ Very recently, $\mathrm{O}^{\prime}$ Connor and colleagues, ${ }^{28}$ reported the case of hypoglycemia detection by a female Cavalier King Charles Spaniel in a 72-year-old non-diabetic man.

Thus far, attempts to demonstrate that dogs can detect hypoglycemia are based on little more than brief anecdotal reports. The present study was therefore designed to explore whether there is any validity to the notion that untrained domestic dogs can detect hypoglycemia in people with type 1 diabetes. This is an important issue with potentially farreaching consequences. If, for example, it is discovered that dogs can detect hypoglycemia reliably, and the mechanisms underlying this feat are established, then it may be possible to develop noninvasive electronic sensor systems to perform the same task. Trained dogs, and/or appropriately designed electronic sensor systems, could then be systematically employed as an early-warning system for people with diabetes, allowing appropriate action to be taken before the effects of hypoglycemia become disabling.

\section{Methods}

\section{Survey}

A purpose-designed survey (available from the lead author upon request), consisting of forced-choice and openended questions, was developed to assess the reported experiences of patients with type 1 diabetes concerning their pet dogs' responses to hypoglycemia. The survey was divided into four sections:
- Section 1 collected information on participant demographics, e.g., sex, age, marital status, occupation, etc.

- Section 2 concentrated specifically on the health of the participants in relation to their diabetes. Questions were posed to collect details on the length of time the participants had suffered from type 1 diabetes, how well controlled they considered their condition to be, and, if known, their latest $\mathrm{HbA1c}$ reading. Since the study was concerned specifically with hypoglycemia detection, categorical-style questions were also constructed to assess the frequency with which the participants experienced hypoglycemic episodes, the types of symptoms normally experienced with such events, and how good the individuals considered their self-awareness of their hypoglycemia-related symptoms to be.

- Section 3 of the survey was concerned with dog ownership, and collected demographic information on the dogs owned by the participants, i.e. sex, age, breed, length of pet ownership.

- Section 4 was designed to collect information on the reaction of the participants' dogs (if any) to their owner's hypoglycemic episodes. Participants were initially asked if their $\mathrm{dog} / \mathrm{s}$ had ever reacted to any of their hypoglycemic episodes (yes, no). Individuals reporting in the positive, were required to answer further categorical questions designed to assess how many episodes of hypoglycemia their dogs had reacted to and the frequency, speed, and reliability with which they typically responded to drops in their owner's sugar levels. The final part of Section 4 allowed participants the opportunity to provide in-depth qualitative information on the behavioral reactions of their dogs to hypoglycemic events.

\section{Procedure}

The survey outlined above was made available both online and in the form of a hardcopy questionnaire. A feature advertising the research and calling for participant recruitment was placed in several UK-distributed newsletters and on diabetes-related websites. The feature provided background information on the research and gave interested parties the opportunity either to complete the survey on the website, or, if preferred, to request a hardcopy version, distributed by post. A covering letter outlining how to complete the survey was made available both online and, for those who opted to complete the paper version of the questionnaire. A freepost return envelope was also provided to this latter category of participants.

It was made clear in both the advertising feature and the covering note that individuals would only be considered eligible for participation if they: (1) had medically diagnosed type 1 diabetes, (2) were 18 years of age or older, and (3) owned one or more dogs.

\section{Data analysis}

Simple descriptive statistics were carried out for each question on the survey. A binary logistic regression analy$\operatorname{sis}^{29}$ was used to explore whether canine sex (male, female), age ( $<6$ months, $6-12$ months, $1-5$ years, $>5$ years), pedigree status (i.e., pedigree vs. crossbreed) and/or length of pet ownership ( $<6$ months, 6-12 months, 1-5 years, $>5$ years) predicted hypoglycemia-response likelihood. The 
qualitative information that owners provided on their dog's behavioral responses to their hypoglycemic episodes was categorized to establish how many dogs were reported to show specific types of reactions.

\section{Ethical approval}

Full ethical permission for the study was granted by the Research Ethics Committee, School of Psychology, Queen's University Belfast.

\section{Results}

\section{Participants}

Two hundred and twelve people chose to participate in the study, $206(97.2 \%)$ via the online survey option and six $(2.8 \%)$ by way of the hardcopy postal questionnaire. Demographic information on the sample can be found in Table 1, which shows that the majority of respondents were female, between 18-45 years of age, married with no children, and in full-time employment.

\section{Participants' health}

Most of the respondents reported having suffered from medically diagnosed type 1 diabetes for longer than 10 years, and they considered their control of the condition to be "quite good" (Table 2). Latest HbA1c readings varied considerably (range: $3.9 \%-18.0 \%$ ) among those people who knew their most recent outputs $(n=146 ; 68.9 \%)$, with a mean

Table 1. Demographic Information ON THE Respondents to THE StUdy

\begin{tabular}{lrc}
\hline Demographic factor & Number & Percentage \\
\hline Sex & & \\
Male & 76 & 35.8 \\
Female & 136 & 64.2 \\
Age (years) & & \\
18-35 & 80 & 37.7 \\
36-45 & 59 & 27.8 \\
46-55 & 42 & 19.8 \\
56-65 & 22 & 10.4 \\
over 65 & 9 & 4.2 \\
Marital status & & \\
Married & 102 & 48.1 \\
Single & 60 & 28.3 \\
Cohabiting & 36 & 17.0 \\
Divorced & 7 & 3.3 \\
Widowed & 7 & 3.3 \\
Children & & \\
0 & 108 & 50.9 \\
1 & 34 & 16.0 \\
2 & 47 & 22.2 \\
3+ & 23 & 10.9 \\
Occupational status & & \\
Employed full time & 96 & 45.3 \\
Unemployed & 33 & 15.6 \\
Employed part time & 30 & 14.2 \\
Student & 26 & 10.4 \\
Retired & 22 & \\
Other & 5 & \\
\hline
\end{tabular}

Table 2. Participants' Responses to Questions Concerning their Diabetes

\begin{tabular}{lrr}
\hline Response & Number & Percentage \\
\hline Duration of illness & & \\
$<1$ year & 12 & 5.7 \\
1-5 years & 40 & 18.9 \\
6-10 years & 21 & 9.9 \\
>10 years & 139 & 65.6 \\
Diabetes control & & \\
Very good & 42 & 19.9 \\
Quite good & 126 & 59.7 \\
Quite poor & 40 & 19.0 \\
Very poor & 3 & 1.4 \\
Frequency of hypoglycemia episodes & & \\
$\quad$ Every day & 13 & 6.1 \\
A few times a week & 99 & 46.7 \\
A few times a month & 48 & 36.8 \\
A few times a year & 22 & 10.4 \\
Hypoglycemia awareness & & \\
Very good & 61 & 28.8 \\
Quite good & 102 & 48.1 \\
Quite poor & 41 & 19.3 \\
Very poor & 8 & 3.8 \\
\hline
\end{tabular}

reading of $7.8 \%$ (95\% confidence interval [CI] 7.4-8.0), slightly above the satisfactory control range of $6.5 \%-7.5 \%$.

\section{Hypoglycemia awareness}

Most respondents reported having symptoms of low blood sugar "a few times a week," and they considered themselves to be "quite good" at recognizing their own symptoms of hypoglycemia (Table 2). Sweating, poor concentration, and trembling were the most commonly reported symptoms of low blood sugar, although a smaller proportion of the sample also indicated experiencing headaches, breathing problems, and seizures (Table 3).

\section{Dog ownership}

Information on the types of dogs owned by the respondents is provided in Table 4. Most of the dogs were male, over one year of age, and had been owned for over 12 months. Seventeen people $(8.0 \%)$ owned more than $1 \mathrm{dog}$. Significantly $(p<0.001$, binomial test) more pedigree dogs were owned than cross-breeds. The pedigree animals comprised 45 different breeds, ranging from large, working breeds (Newfoundlands, German Shepherds, Border Collies) through to smaller, toy breeds (Chihuahuas, Bichon Frisé, Yorkshire Terriers). The most commonly kept breeds were Labrador Retrievers (owned by 23 of the respondents), Border Collies $(n=14)$, and Golden Retrievers $(n=10)$.

\section{Dogs and hypoglycemia awareness}

Respondents were more likely than not to report that their dogs showed hypoglycemia awareness, i.e., that they had reacted to a hypoglycemic episode on at least one occasion (138 [65.1\%] versus 74 [34.9\%]; $p<0.001$, binomial test). Binary logistic regression analysis showed that canine sex, age, pedigree status, and length of pet ownership did not significantly 
TABle 3. Symptoms of Hypoglycemia REPORTED BY THE RESPONDENTS

\begin{tabular}{lcc}
\hline Symptom & Number & Percentage \\
\hline Sweating & 143 & 67.5 \\
Poor concentration & 124 & 58.5 \\
Trembling & 113 & 53.3 \\
Confusion & 109 & 51.4 \\
Irritability & 106 & 50.0 \\
Disorientation & 97 & 45.8 \\
Poor coordination & 89 & 42.0 \\
Hunger & 85 & 40.1 \\
Blurred vision & 81 & 38.2 \\
Weakness & 78 & 36.8 \\
Tiredness & 78 & 36.8 \\
Heart palpitations & 71 & 33.5 \\
Dizziness & 67 & 31.6 \\
Speech problems & 54 & 25.5 \\
Anxiety & 47 & 22.2 \\
Nervousness & 38 & 17.9 \\
Headaches & 32 & 15.1 \\
Loss of consciousness & 22 & 10.4 \\
Breathing problems & 14 & 6.6 \\
Seizures /fits & 14 & 6.6 \\
Coma & 1 & 0.5 \\
\hline
\end{tabular}

$(p>0.05)$ predict a dog's propensity to respond to its owner's episodes of hypoglycemia (Table 5). The Hosmer and Lemeshow test demonstrated that the model adequately fitted the data $\left(\chi^{2}=3.86, d f=7, p=0.79\right)$.

Most of the dogs who were reported to have responded to their owner's "lows" had done so several times, and in response to most of the known hypoglycemic episodes (Table 6). The majority of dogs were also reported to respond to these episodes "quite early on," i.e., in the early stages of the person realizing they were hypoglycemic. Just over a third of the dogs were believed to have shown behavioral responses before the owners themselves were aware they were hypoglycemic. Owners indicated that their dogs showed "alert" behavior in a wide variety of contexts, ranging from exercising to sleeping (Table 6).

The nature of the dogs' behavioral responses to their owners' hypoglycemic episodes varied between animals. Most of the dogs were perceived by their owners to engage in behaviors aimed at attracting the owner's attention. Thus, many were reported to nudge or nuzzle their owners with their noses $(n=56 ; 40.6 \%)$, jump on top of them $(n=42$; $30.4 \%)$ and / or lick at their hands $(n=30 ; 21.7 \%)$ and faces $(n=38 ; 27.5 \%)$, particularly around the mouth.

Vocalizations, including whining $(n=45 ; 32.6 \%)$, barking $(n=34 ; 24.6 \%)$, and howling $(n=6 ; 4.3 \%)$ were commonly reported. Many participants $(n=53 ; 38.4 \%)$ also indicated that their dogs became agitated, with some pacing back and forth or jumping about. Other dogs appeared calmer, either sitting directly in front of the owner $(n=14 ; 10.1 \%)$ or following the owner, almost like a shadow $(n=54 ; 39.1 \%)$. Reports of dogs staring intently at the person's face were relatively common $(n=57 ; 41.3 \%)$.

Twenty-eight people (20.3\%) indicated specifically that their dogs woke them up from sleep (usually by displaying one or more of the preceding behaviors); in most of these cases $(89.3 \%)$ the animal slept in an entirely different room, and some dogs $(n=5 ; 17.8 \%)$ had to scratch or paw at a closed door before gaining entry to the bedroom.

A smaller number of dogs showed behaviors somewhat suggestive of fear, including trembling $(n=10 ; 7.2 \%)$, running into a different room $(n=7 ; 5.1 \%)$, and/or panting $(n=3 ; 2.2 \%)$. Some owners $(n=15 ; 10.9 \%)$ also indicated that their dogs tried to attract the attention of, and/or fetch, someone else in the house. One woman indicated that her dog had bitten her on the face after she had fallen into a diabetic coma.

None of the people who owned two or more dogs $(n=$ 17 ), indicated that more than one of their pets showed behavioral responses to their hypoglycemic episodes.

\section{Discussion}

The findings from this study suggest that behavioral reactions to hypoglycemic episodes in pet owners with type 1 diabetes commonly occur in untrained domestic dogs. Most of the respondents to this survey reported owning a dog that showed behavioral responses to hypoglycemia, and the frequency and reliability with which these animals alerted their owners to hypoglycemic episodes was relatively high. Over a third $(36.0 \%)$ of the owners in the sample believed that their dogs reacted most times they went low, with the majority of animals (40.8\%) showing behavioral responses at roughly the same time as the owners themselves were aware they were hypoglycemic. Moreover, just over a third (33.6\%) of the dogs showed reactions before their owners had realized they were low, prompting appropriate action on the part of the owner (e.g., glucose readings, food consumption).

A wide variety of dogs were represented by the participants to the survey in terms of breed, sex, and age. Analysis revealed no significant relationships, however, between canine demographics and hypoglycemia response likelihood. Thus, owners of Poodles and Bichon Frisés were just as likely to report behavioral reactions to their hypoglycemic episodes as the keepers of German Shepherds and Labrador Retrievers. This is a positive finding in light of how difficult

Table 4. Demographic Information on THE RESPONDENTS' DOGS

\begin{tabular}{lrc}
\hline Demographic factor & Number & Percentage \\
\hline Sex & & \\
$\quad$ Male & 118 & 55.7 \\
$\quad$ Female & 94 & 44.3 \\
Age & 3 & \\
$\quad<6$ months & 15 & 1.4 \\
7-12 months & 99 & 7.1 \\
1-5 years & 95 & 46.7 \\
$\quad$ 5 years & & \\
Length of time owned & 11 & 5.8 \\
$\quad<6$ months & 11 & 5.2 \\
7-12 months & 95 & 44.8 \\
1-5 years & 95 & 70.2 \\
$\quad$ 5 years & & 29.8 \\
Breed status & 146 & \\
$\quad$ Pedigree & 62 & \\
$\quad$ Mongrel & & \\
\hline
\end{tabular}


Table 5. Results of Binary logistic Regression Analysis for Hypoglycemia-Response Likelihood, with Predictor Variables of Dog Sex, Age, Breed Status, and Length of Pet Ownership

\begin{tabular}{lccc}
\hline Variable & & $\begin{array}{c}\text { Exp }(B) \\
95 \% \text { CI }\end{array}$ & p Value \\
\hline Dog sex & Odds ratio & $0.50-1.65$ & 0.76 \\
Dog age & 0.91 & $0.03-13.76$ & 0.61 \\
Dog age & & $0.40-9.30$ & 0.77 \\
Dog age & 0.64 & $0.65-4.29$ & 0.41 \\
Dog age & 1.93 & $0.83-3.27$ & 0.28 \\
Breed status & 1.67 & $0.33-13.57$ & 0.16 \\
Length of ownership & 1.64 & $0.36-8.69$ & 0.50 \\
Length of ownership & & $0.28-1.86$ & 0.42 \\
Length of ownership & 2.13 & & 0.48 \\
Length of ownership & 1.76 & 0.51 \\
Constant & 0.73 & 0.32 & 0.003 \\
\hline
\end{tabular}

CI, confidence interval.

the selection and recruitment of dogs for training purposes can be. ${ }^{16,20}$

The reported behavioral reactions of dogs to their owner's hypoglycemic episodes differed between animals. Most dogs $(64.5 \%)$ were reported to behave in a manner suggestive of attracting their owners' attention, for example, by jumping up toward the owner's face, licking the owner, and/or barking. Many of these dogs acted in ways that reduced the physical distance between themselves and their owners, with some animals attempting to sit on top of their caregivers or following them around like a shadow; increased eye contact, with dogs looking at their owners more frequently, also appeared to be relatively common.

A smaller proportion of dogs were perceived by their owners to be quite frightened by their blood sugar drops, moving themselves into another room, trembling, and/or hyperventilating. Interestingly, all of the multiple dog-owning participants, reported that only one of their pets showed "alert" behavior. This implies that not all dogs are reactive to the cues that signal hypoglycemia, even when emitted by the same individual.

Table 6. Participants' Responses to Questions Concerning Their Dogs' Reactions to Their Owner's Hypoglycemic Episodes

\begin{tabular}{|c|c|c|}
\hline Response & Number & Percentage \\
\hline \multicolumn{3}{|l|}{ Number of hypoglycemic episodes } \\
\hline 1 & 35 & 25.4 \\
\hline $2-5$ & 42 & 30.4 \\
\hline $6-10$ & 17 & 12.3 \\
\hline $11+$ & 44 & 31.9 \\
\hline \multicolumn{3}{|l|}{ Frequency of reactions } \\
\hline Every time & 22 & 16.2 \\
\hline Most times & 49 & 36.0 \\
\hline Only occastionally & 32 & 23.5 \\
\hline Hardly ever & 33 & 24.3 \\
\hline \multicolumn{3}{|l|}{ Speed of detection } \\
\hline $\begin{array}{l}\text { Very early on } \\
\text { (before the owner is aware he/she is } \\
\text { becoming hypoglycemic) }\end{array}$ & 42 & 33.6 \\
\hline $\begin{array}{l}\text { Quite early on } \\
\text { (in the early stages of the owner's realizing } \\
\text { that he/she is hypoglycemic) }\end{array}$ & 51 & 40.8 \\
\hline $\begin{array}{l}\text { Quite late on } \\
\text { (after the owner has taken action to raise } \\
\text { sugar levels) }\end{array}$ & 24 & 19.2 \\
\hline $\begin{array}{l}\text { Very late on } \\
\text { (after the owner has lost consciousness) }\end{array}$ & 8 & 6.4 \\
\hline \multicolumn{3}{|l|}{ Activities engaged in by owner } \\
\hline Resting & 55 & 31.4 \\
\hline Moving about & 52 & 29.7 \\
\hline Sleeping & 49 & 28.0 \\
\hline Exercising & 19 & 10.9 \\
\hline
\end{tabular}


This study was purely concerned with elucidating dogs' reactions to hypoglycemia in people with type 1 diabetes. That said, a few people with type 2 diabetes $(n=14)$ also contacted the investigators, indicating that their dogs reliably showed "alert" behavior. Likewise, five owners of cats reported the same phenomenon, in all cases waking their owners up from nocturnal episodes of hypoglycemia. Further work is needed to explore the range of animal species that might be responsive to changes in the diabetic state.

The findings from this investigation lead one to question what signals dogs might be detecting in their hypoglycemic owners. Odor cues appear, at first glance, to be the most plausible explanation. Chen and colleagues, ${ }^{26}$ for example, found that one dog reportedly exhibited "alert" behavior when its owner was asleep, and possibly emitting no cues other than olfactory ones. Similar findings were shown by this survey. Indeed $23.1 \%$ of the participants reported that their dog had, on at least one occasion, woken them up from a nocturnal "low." In most of these cases the dog slept in another room of the house, and in some situations the door of the owner's bedroom was closed, forcing the animal to scratch or paw at the obstacle.

Increases in sweating have been repeatedly noted in hypoglycemic individuals. ${ }^{21}$ As in the case of cancer, it is possible that dogs can detect changes in the chemical composition of their owners' sweat, using their acute sense of smell. However, it cannot be ignored that dogs may respond to other cues besides olfactory ones, including, for example, subtle changes in their owner's mood (with people often becoming more irritable as their sugar levels drop) or visual signals related to their owner's behavior (with some people trembling, becoming disorientated, losing consciousness, or having seizures). The possibility that individual animals employ multiple signals, or that different dogs use entirely different cues equally cannot be dismissed. Research is required to determine what types of cue/s dogs employ to detect hypoglycemia. By establishing what it is that dogs are reacting to, it will it be possible to train such animals to alert in the correct manner.

Although not eligible for participation in this investigation, a female owner of a trained hypoglycemia alert dog, reported an inability of the animal to detect decreases in her sugar levels while she slept, presumably because the animal had only been trained to alert to subtle visual changes in behavior associated with her sugar level's dropping; in this case rubbing of her hands over her face was the visual cue.

This investigation benefits from having used a relatively large cohort of people with type 1 diabetes, all with firsthand experience of their pets' reactions to their hypoglycemic episodes. The participants, however, represented a self-selected sample. People owning dogs with a propensity to show overt "alert" behaviors, may thus have been more inclined to take part in the study than those whose pets showed little or nothing in the way of a behavioral response. That said, a published abstract of a questionnaire-based study conducted in Australia indicated that 72 of 106 people with diabetes whose dogs had witnessed a hypoglycemic event, reported behavioral reactions in their pets to their "lows." 30 Although the methods from this abstract are unclear, taken together, these two studies provide convincing evidence for a proportion of dogs being able to spontaneously detect hypoglycemia-related cues in their owners.
Some dog owners may not be attuned to their pets' reactions to their hypoglycemic episodes, which may have underestimated the number of dogs that can detect low blood sugar. In light of this, it is difficult to draw finite conclusions on what proportion of the canine population may be reactive to changes in their owners' diabetic states, except to say that this is a commonly reported phenomenon.

The present study provides a useful basis for further, more controlled experimentation in this area. A prospective, longitudinal approach, that uses owners' blood glucose readings as confirmation of hypoglycemia detection by dogs, could yield objective and valuable data and would complement the more cross-sectional, retrospective design adopted here. The prospect of continued work in this area will advance our understanding of the value of domestic dogs as an alerting mechanism for people suffering from diabetes and other chronic conditions, e.g., epilepsy.

\section{Conclusions}

The findings from this study suggest that many dogs can detect hypoglycemia, often without the use of visual cues and before the animals' caregivers are aware of their own symptoms. Although it was not the goal of this project to explore how dogs detect hypoglycemia, the results hint at an odor cue, although other signals (e.g., changes in owner behavior due to impaired cognitive functioning) cannot be dismissed. Research is required to elucidate what mechanism/s might underlie the ability of dogs to detect hypoglycemia and to determine whether animals can be trained to consistently alert their owners to the onset of hypoglycemia.

\section{Acknowledgments}

The authors thank Diabetes UK for funding this research, and all of those individuals who participated in the study. Thanks also to Geoff Caves, School of Psychology, Queen's University Belfast, for his help with the online survey. The corresponding author is an employee of Queen's University Belfast; both of the co-authors are employees of the University of Lincoln. None of the authors have any competing interests to declare.

\section{Disclosure Statement}

No competing financial interests exist.

\section{References}

1. Pet Food Manufacturer's Association. Profile 2007. PFMA 2008.

2. Friedmann E, Thomas SA, Eddy TJ. Companion animals and human health: Physical and cardiovascular influences. In: Podberscek A, Paul ES, Serpell JA, eds. Companion Animals and Us. Cambridge: Cambridge University Press, 2000:125-142.

3. Hart LA. Community context and psychosocial benefits of animal companionship. In: Fine A, ed. Handbook of Animal-Assisted Therapy. London: Academic Press, 2006:73-94.

4. McNicholas J, Gilbey A, Rennie A, Ahmedzai S, et al. Pet ownership and human health: A brief review of evidence and issues. BMJ 2005;331:1252-1255.

5. Wells DL. Domestic dogs and human health: An overview. Br J Health Psychol 2007;12:145-156. 
6. Wilson CC, Turner DC. Companion Animals in Human Health. London: Sage Publications, 1998.

7. Williams H, Pembroke A. Sniffer dogs in the melanoma clinic? Lancet 1989;i:734.

8. Church J, Williams H. Another sniffer dog for the clinic? Lancet 2001(9285);358:930.

9. Dobson R. Dogs can sniff out first signs of men's cancer. Sunday Times 2003;27th April.

10. Fraser L. Scientists put sniffer dogs on the scent of men with cancer. Sunday Telegraph 2002;2nd June.

11. Willis CM, Church SM, Guest CM, Cook WA, et al. Olfactory detection of human bladder cancer by dogs: Proof of principle study. BMJ 2004;329:712-716.

12. Leahy M. Olfactory detection of human bladder cancer by dogs: Cause or association? BMJ 2004;329:1286.

13. Pickel D, Manucy GP, Walker DB, Hall SB, et al. Evidence for canine olfactory detection of melanoma. Appl Anim Behav Sci 2004;89:107-116.

14. McCulloch M, Jezierski T, Broffman M, Hubbard A, et al. Diagnostic accuracy of canine scent detection in early- and late-stage lung and breast cancers. Integr Cancer Ther 2006;5:30-39.

15. Brown SW, Strong V. The use of seizure-alert dogs. Seizure 2001;10:39-41.

16. Dalziel DJ, Uthman BM, McGorray SP, Reep RL, et al. Seizure-alert dogs: A review and preliminary study. Seizure 2003;12:115-120.

17. Kirton A, Wirrell E, Zhang J, Hamiwka L, et al. Seizure-alerting and -response behaviors in dogs living with epileptic children. Neurology 2004;62:2303-2305.

18. Strong V, Brown SW, Walker R. Seizure-alert dogs-fact or fiction. Seizure 1999;8:62-65.

19. Strong V, Brown S, Huyton M, Coyle H. Effect of trained Seizure Alert Dogs on frequency of tonic-clonic seizures. Seizure 2002;11:402-405.

20. Strong V, Brown SW. Should people with epilepsy have untrained dogs as pets? Seizure 2000;9:427-430.
21. McAulay V, Deary IJ, Frier BM. Symptoms of hypoglycaemia in people with diabetes. Diabet Med 2001;18:690-705.

22. Smith D, Amiel SA. Hypoglycaemia unawareness and the brain. Diabetologia 2002;45:949-958.

23. Aussedat B, Thome-Duret V, Reach G, Lemonnier F, et al. A user-friendly method for calibrating a subcutaneous glucose sensor-based hypoglycaemic alarm. Biosens Bioelectron 1997;12:1061-1071.

24. Harris ND, Ireland RH, Marques JLB, Hudson S, et al. Can changes in QT interval be used to predict the onset of hypoglycemia in type 1 diabetes. Comput Cardiol 2000;27: 375-378.

25. Lim K, Wilcox A, Fisher M, Burns-Cox CJ. Type 1 diabetics and their pets. Diabet Med 1991;9:S3-S4.

26. Chen M, Daly M, Williams N, Williams S, et al. Non-invasive detection of hypoglycaemia using a novel, fully biocompatible and patient friendly alarm system. BMJ 2000;321: 1565-1566.

27. Tauveron I, Delcourt I, Desbiez F, Somda F, et al. Canine detection of hypoglycaemic episodes whilst driving. Diabet Med 2006;23:335.

28. O'Connor MB, O'Connor $\mathrm{C}$, Walsh $\mathrm{CH}$. A dog's detection of low blood sugar: A case report. Ir J Med Sci 177:155-157.

29. Brace N, Kemp R, Snelgar R. SPSS for Psychologists. Hampshire: Palgrave Macmillan, 2003.

30. Stocks AE. Can dogs help patients with hypoglycaemia? Diabetologia 2002;45:A79.

Address reprint requests to: Deborah L. Wells, Ph.D. School of Psychology Queen's University Belfast Belfast BT7 1NN Northern Ireland United Kingdom

E-mail: d.wells@qub.ac.uk 